\title{
Spongy, Nongravid Uterine Fibroid Due to Hydropic Change: Case Report and Review
}

\section{Yahia F Dajani $^{*} \mid$ Ali M Akilah ${ }^{2}$}

*Correspondence: Yahia F Dajani

Address: ${ }^{1}$ Grand Lab, Jabal Amman, Amman, Jordan; ${ }^{2}$ Akilah Hospital, Jabal Amman, Amman, Jordan

e-mail $\bowtie$ : medlab@go.com.jo

Received: 09 March 2021; Accepted: 15 March 2021

Copyright: (C) 2021 Dajani YF. This is an open-access article distributed under the terms of the Creative Commons Attribution License, which permits unrestricted use, distribution, and reproduction in any medium, provided that the original work is properly cited.

\section{ABSTRACT}

We report unique regressive changes in a large, non-gravid uterine leiomyoma of a 32 year old lady who, before 2015, had had seven recurrent spontaneous, alternating with assisted pregnancies, over a 7-year period (2008-1015 AD). In formalinfixed status, cut surface of the leiomyoma showed a sponge-like appearance. Pathogenesis of resultant degenerative changes in respect of repeated hormonal hits is discussed, together with review of the literature. To our knowledge, such extreme multi-cystic change in leiomyoma with spongy appearance has not been previously reported.

Keywords: Non-Gravid Uterine Leiomyoma, Multi-Cystic Change, Diverse Hormonal Hits

\section{Introduction}

Unprecedented degenerative change with sponge like appearance of a uterine leiomyoma is reported in a lady who had repeated pregnancies, spontaneous and assisted, over a 7-year period. It is proposed that causation of this quaint effect on leiomyoma was related to diverse hormonal hits, added to formalin fixation artifacts developing during the short post-operative period. This case adds to the catalog of degenerative changes that may be seen in uterine leiomyomas. Review of the subject is presented.

\section{Case Report}

History: A 32 year old lady, Para 3+5, presented to our clinic in May 2019 with irregular, heavy vaginal bleeding. Hemoglobin was 8g/dl, Hematocrit $26.1 \mathrm{l} / \mathrm{l}$, Red cell count 3.48 10^12/l, MCV 75fl, MCH $23 \mathrm{pg}$, MCHC 31g/dl, White cell count $11.50010^{\wedge} 9 / \mathrm{l}$ with normal differential, Platelet count 421.000 $10^{\wedge} 9 / 1$.

She gave a past history of seven pregnancies as follows: In 2008 she had an early miscarriage after IUI (Intra-uterine insemination) and Clomid; in 2009 spontaneous pregnancy with early miscarriage; again in 2009, spontaneous molar pregnancy for which she underwent suction and curettage. In 2011 by IVF (in-vitro fertilization) she had a single live male baby. Frozen embryos extracted from that very cycle 
replaced later failed. In 2013, she had spontaneous pregnancy with early miscarriage. In 2014, she had spontaneous pregnancy with early miscarriage. In 2015, she had her last (seventh) pregnancy by IVF and delivered twins by Caesarian section, one male and one female.

Speculum vaginal examination was normal. She gave a history of two episodes of deep vein thrombosis with recovery, in 2011 and 2015 respectively.

An ultra-sonogram showed an intrauterine multi-cystic mass $8 \mathrm{~cm}$ in diameter (Fig. 1). Hysteroscopy showed a normal uterine cavity. Endometrial biopsy showed postovulatory phase type endometrium and was otherwise unremarkable. In view of the symptomatic and large intra-uterine mass, a total abdominal hysterectomy was performed 10 days later. On the operation table, a large uterine mass showed unusual cystic change (Fig. 2). Postoperative course was uneventful and she was discharged on the second postoperative day in a good condition.

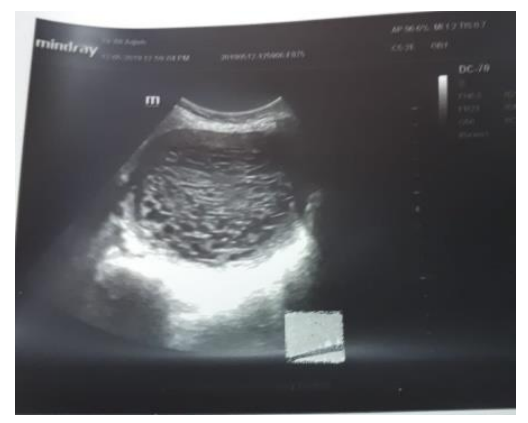

Figure 1: Ultrasonogram showing uterine cystic mass

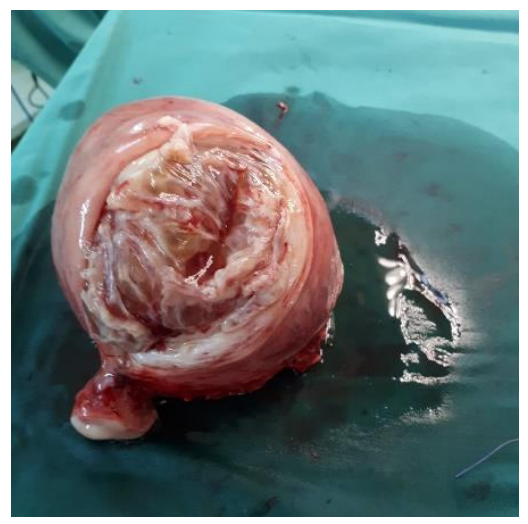

Figure 2: Intra-operative. Uterine fibroid with widespread cystic change

\section{Pathological Examination}

Gross Examination: A $692 \mathrm{~g}$, round uterus with protruding cervix $3 X 2.5 \mathrm{~cm}$. After 24-hour formalin fixation, cut surface showed a well defined mucoid intra-mural mass $8 \mathrm{~cm}$ in diameter with striking sponge like appearance (Fig. 3). Opposing uterine wall was $1.5 \mathrm{~cm}$ thick. 


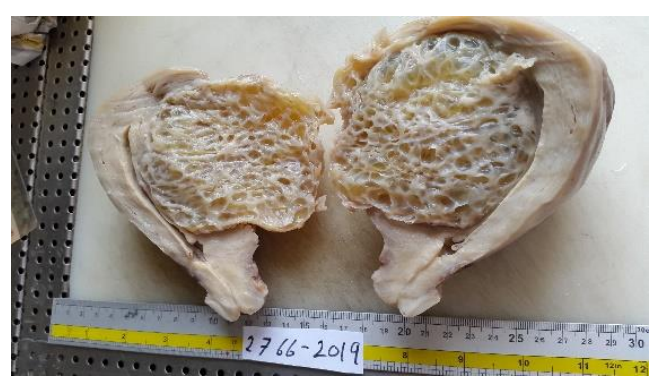

Figure 3: Uterine fibroid. Post formalin sponge appearance

Microscopic Examination: Non-secretory endometrium with cervix showing benign squamocolumnar junction. The intramural mass showed a well defined interface with myometrium, appeared multi-cystic, sponge-like, the cysts exhibiting a concave and flattened lining (Fig. 4). Inter-cystic mantles were filled by loose to moderately cellular, interlacing bundles of smooth muscle fibers which reacted to both Desmin (Fig. 5) and Smooth Muscle Actin (Fig. 6). Component cells showed ovoid nuclei with mild pleomorphism, apoptosis and mitoses averaging 0.8 per 10 high power (X40) fields with a range of 0-2. There was sparse permeation by lymphocytes and mast cells with occasional lymphocytic aggregate seen. There was no evidence of coagulative necrosis, hemorrhage or anaplasia. Content of cystic spaces proved negative for Alcian blue, mucicarmine and PAS (Periodic Acid Schiff).

Pathological Diagnosis: $8 \mathrm{~cm}$ Intramural uterine leiomyoma with striking spongy appearance, presumably due to degenerative change. no evidence of malignancy.

Twenty months postoperatively, the patient is well and symptom free.

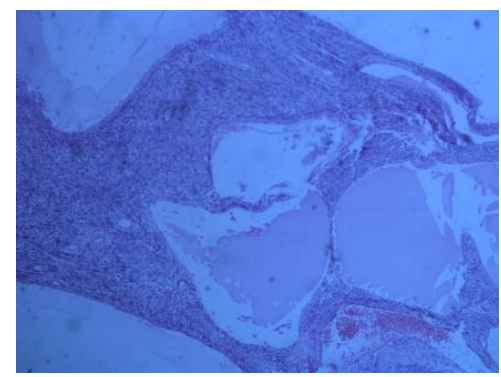

Figure 4: X4 HE 2766-2019 Multiple small cysts among muscle fibers

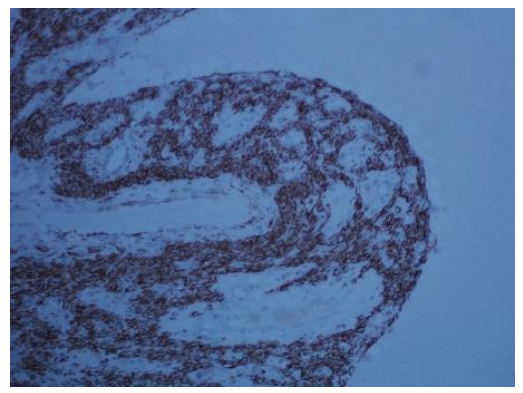

Figure 5: X20 Desmin 2766-2019 


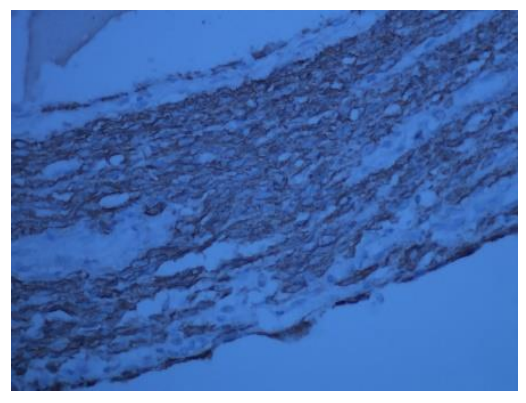

Figure 6: x40 ACTIN

\section{Discussion}

Uterine smooth muscle tumors are among the most common gynecological lesions, presenting in a great variety of ways, both in the clinical and pathological settings. They may remain silent for a long time or, alternatively, cause serious consequences such as menorrhagia or infertility.

In the pathology literature, several treatises have been devoted to wide variations in the morphology of smooth muscle tumors. In one report, they were divided into non-degenerate or degenerate forms (cystic, hemorrhagic, hyaline, fatty, myxoid), also variant forms such as mitotically active, cellular, atypical or STUMP (Smooth Muscle Tumor of Undetermined Potential), the malignant counterpart being leiomyosarcoma (Kagan Arleo et al., 2015). Others have described morphological variants and difficulties encountered sometimes, including cellular, atypical, epithelioid leiomyomas as well as those with secondary changes such as hemorrhage, infarction, myxoid and perinodular hydropic changes (Abraham and Saldanha, 2013). Hydropic change corresponds to accumulation of fluid within the lesion which occurs in selective zones. Extensive hydropic change had been reported in pregnancy, proposed to be due to pressure effect on venous return. Diffuse or extensive hydropic change in leiomyoma was also reported in nongravid uterus (Horta et al., 2015; Patil et al., 2018). However, perinodular hydropic change, described by others, was not seen in our case (Jashnani et al., 2010). Massive multilocular degeneration was also reported as extremely rare (Coard and Plummer, 2007). Hemangiomatous elements have been reported in the uterus, but cavernous hemangiomatous lesion developing in an intramural uterine leiomyoma is extremely rare (Celik et al., 2017). Histology of our case did not even remotely resemble the case described in this last aforementioned report. In another report, submucosal leiomyoma with degeneration, among 8 miscellaneous lesions, was described as causing thick endometrium with honeycomb appearance discovered by transvaginal sonographic on hysteroscopy (Lee and Park, 2017). In this report, the lesion was sub-endometrial with no pathological description in contrast to the large intramural lesion in our case.

From our files, we found a severe case of cystic change with beehive effect in a case of adenomyosis 
uteri (Fig. 7). In contrast to our present case, the cystic spaces were less homogenous and occurred in the glandular component of endometrial rests, not in smooth muscle (Fig. 8). To our knowledge, nothing like the gross and histological changes in the present case has been reported to date.

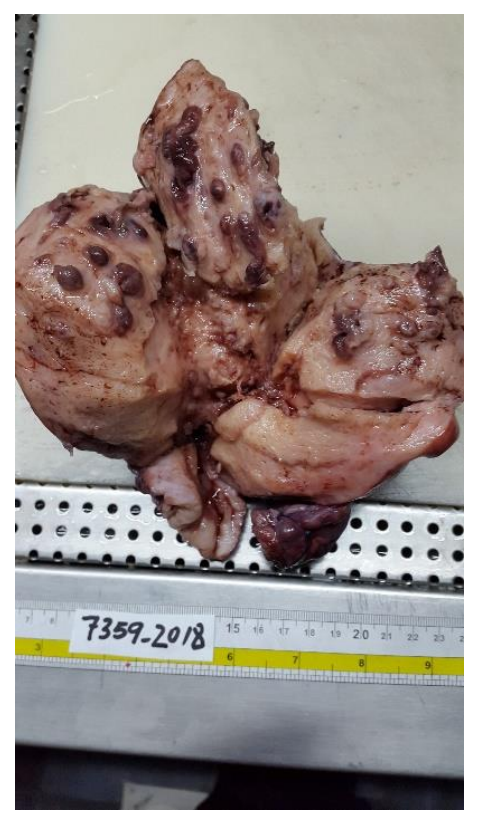

Figure 7: Adenomyoma with honeycomb change

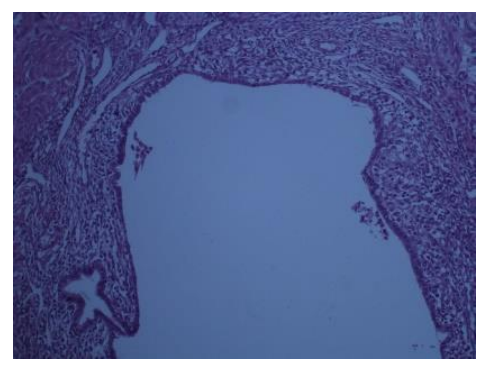

Figure 8: X20 Intraendometrial cystic change in adenomyosis

Treatment options include myomectomy, conservative methods or hysterectomy (Laughlin and Stewart, 2011). In our case, the size of tumor and presenting symptoms justified hysterectomy, especially that there was no intention of bearing more children.

In our present case, recurrent exposure of the intra-uterine leiomyoma to hormonal stimulation causing perturbations in its internal milieau might have been operative in the degenerative changes found at hysterectomy. Leiomyomas proliferate slowly and usually enlarge through expansion of interstitium by producing interstitial collagens and proteoglycans. Thus, medical treatment of symptomatic leiomyomas should aim at dissolving extracellular matrix as much as inhibiting cell proliferation. Several conservative approaches have been sought which have not been entirely successful in fulfilling this aim. In this context. Non-Steroidal Anti-inflammatory Drugs or NSAIDS (ibuprofen, 
naproxen, and mefenamic acid) were used to check menorrhagia by inhibiting the enzyme cyclooxygenase to reduce prostaglandins. Although effective, NSAIDS are less so than Tranexamic acid in controlling menorrhagia, but neither is known to dissolve leiomyoma matrix. Tranexamic acid is a synthetic agent that prevents fibrin degradation, therefore favors clotting, necrosis and infarction of leiomyomas. But Tranexamic acid has not proved effective in reducing matrix and size of uterine leiomyomas. $17 \beta$-estradiol and progesterone, in combination or progesterone alone, are commonly used to regulate heavy menses irrespective of presence or absence of uterine leiomyomas. Such approach provides only short-term relief, in many cases ending with hysterectomy. The slow-release IUD LNG-IUD (levonorgestrel) suppresses estrogen with thinning of endometrium but bears no direct effect on leiomyoma. Aromatase (CYP19) enzyme converts testosterone to $17 \beta$-estradiol, but there is no evidence on its efficacy in treatment of uterine leiomyomas. GnRH Agonist acts on GnRH (gonadotropin-releasing hormone) that induces gonadotropins release from pituitary, which in turn stimulates production of $17 \beta$ estradiol and progesterone from the ovaries. GnRH agonists (Leuprolide acetate, Goserelin acetate, and Nafarelin acetate) have been used as pre-surgical therapy for symptomatic leiomyomas, inducing a state of hypoestrogenism through downregulation of the hypothalamic-pituitary-ovarian axis, amenorrhea, amelioration of menorrhagia and quick reduction in leiomyomata volume. However, side effects such as myalgia, arthralgia, change of mood, visual impairment and bone loss on long term therapy limit their use. GnRH antagonists (Cetrorelix acetate,Gganirelix acetate, and Nal-Glu), similar to GnRH agonists, can reduce leiomyoma volume via induction of a hypoestrogenic state. But GnRH antagonists are injections and must be taken 2 to 7 times a week, since long-acting depot preparation is not easily accessible. SPRMS (Selective progesterone receptor modulators) include Telapristone acetate, Asoprisnil and Ulipristal acetate, since Progesterone receptor A and B (PR-A, PR-B) protein is elevated within leiomyoma and is implicated in production of extracellular matrix. Modulators of progesterone receptor were discontinued in one trial due to hepatotoxicity, besides risk of endometrial pathology on long term use. Thus, ideal medical treatment for leiomyomas is yet to be discovered. All the aforementioned approaches in recent years of conservative treatment focus on the vulnerability of leiomyomas to hormonal manipulation (Lewis et al., 2018).

\section{Conclusion}

A case of extreme cystic change in a uterine leiomyoma is presented with a sponge like appearance on the cut surface after formalin fixation. Pathogenesis of this degenerative change is discussed in a 32year old lady who had frequented assisted fertility centers over a 7-year period seeking progeny. Unprecedented regressive change seen in our case is discussed in light of possible overly humoral influence on interstitium of leiomyomas. 


\section{References}

Abraham J and Saldanha P. Morphological variants and secondary changes in uterine leiomyomas. International Journal of Biomedical Research 2013; 4: 638-664.

Celik ZE, Bastoklu S, Tuyan Ilhan T and Celik C. Cavernous hemangioma arising in an intramural leiomyoma. Integrative Cancer Science and Therapeutics 2017; 4 (1): 1-3.

Coard K and Plummer J. Massive multilocular cystic change of the uterus. An extreme example of hydropic degeneration. South Medical Journal 2007; 100: 309-312.

Horta M, Cunha TM, Oliveira R, Magro P. Hydropic leiomyoma of the uterus presenting as a giant abdominal mass. BMJ Case Reports 2015; 2015: bcr2015211929.

Jashnani KD, Kim S, Dhamija G. Letter to the editor. Perinodular hydropic degeneration in leiomyoma: An alarming histology. Indian Journal of Pathology \& Microbiology 2010; 53: 173-175.

Kagan Arleo E, Schwartz PE, Hui P, McCarthy S. Review of leiomyoma variants. American Journal of Roentgenology 2015; 205: 912-921.

Laughlin SH and Stewart EA. Uterine Leiomyomas. Individualizing the Approach to a Heterogeneous Condition. Obstetrics and Gynecology 2011; 117: 396-403.

Lee S and Park M. Thick uterine endometrium with "honeycomb" appearance in transvaginal ultrasonography revealed various gynecologic conditions: presented with flexible hysteroscopic findings. Abstract. In: Ultrasound in Obstetrics \& Gynecology. 27th World Congress on Ultrasound in Obstetrics \& Gynecology, Vienna 2017; 50: 16-19.

Lewis TD, Malik M, Britten J, San Pablo AM, Catherino WH. A comprehensive review of the pharmacologic management of uterine leiomyoma. BioMedical Research International 2018; pp: 1-11.

Patil AR, Nandikoors S, Padilu R. Hydropic degeneration of leiomyoma in nongravid uterus: The 'split fiber' sign on MRI. Indian J of Radiological Imaging 2018; 28: 182-186. 